\title{
Gait Parameters Change Prior to Freezing in Parkinson's Disease: A Data-Driven Study with Wearable Inertial Units
}

\author{
Maria Laura Ferster, Sinziana Mazilu, and Gerhard Tröster \\ Wearable Computing Laboratory \\ ETH Zürich, Switzerland \\ \{mazilus, troester\}@ife.ee.ethz.ch
}

\begin{abstract}
Freezing of gait (FoG) is a motor impairment among patients with advanced Parkinson's disease which is associated with falls and has a negative impact on a patient's quality of life. Wearable systems have been developed to detect FoG and to help patients resume walking by means of rhythmical cueing. A step further is to predict the FoG and start cueing a few seconds before it happens, which might help patients avoid the gait freeze entirely.

We characterize the gait parameters continuously with up to 10-12 seconds prior to FoG, observe if and how they change before subjects enter FoG, and compare them with the gait before turns. Moreover, we introduce and discuss new frequency-based features to describe gait and motor anomalies prior to FoG. Using inertial units mounted on the ankles of 5 subjects, we show specific changes in the stride duration and length with up to four seconds prior to FoG on all subjects, compared with turns. Moreover, the dominant frequency migrates towards $[3,8] \mathrm{Hz}$ band with up to six seconds prior to FoG on 3 subjects. These findings open the path to real-time prediction of FoG from inertial measurement units.
\end{abstract}

\section{Keywords}

prediction, wearable sensors, Freezing of Gait, Parkinson's disease, gait parameters, motor impairment analysis

\section{INTRODUCTION}

Parkinson's disease (PD) is a neurological degenerative disorder with a worldwide prevalence of 16.1 million people [16], characterized by rigidity, tremor, reduced movement range and walking difficulties. A common symptom in PD is the so-called freezing of gait (FoG), an impromptu incapacity to walk [19], despite the intention of the subject to do so. Patients have the impression that their feet are glued to the ground [19] and loose control over their gait. FoG is transient and last for a few seconds up to 1-2 minutes [19]. It is the main cause of falls [3] and mortality in PD [6].
There is no cure at the moment for PD, medication being used to milden or temporary release the symptoms. FoG is one of the least responsive to the medical treatment. However, clinical studies suggest that subjects with FoG respond positively to rhythmical external cues, by exiting FoG and resuming natural gait $[5,18]$. Continuous cueing wears of with time [18], thus rhythmic stimulation is given only during FoG and few seconds after, until the patient reestablishes the gait. On-body wearable sensors are used to detect the onset of FoG in real-time [11], and to start temporary rhythmical cueing upon gait freeze [2]. While such systems might be beneficial in shortening the FoG duration [2], they cannot help the user to avoid freeze, since they need at least some hundreds of milliseconds to react to the existent FoG [11]. A step further would be to predict that freeze episodes might happen in the next few seconds and start cueing, thus helping subjects to maintain natural gait and avoid the FoG altogether.

Plotnik and colleagues [21] suggest that FoG is a result of multiple gait impairments, that develop over time, decreasing the gait performance up to the point of freezing. We follow this hypothesis and aim to characterize whether there are specific changes in the gait properties during the transition from normal walking to freeze, and are different to gait variability before other walking events such as turns.

We contribute the following:

(1) We use data collected from inertial measurement units (IMU) mounted on both ankles of 5 subjects with PD and FoG, and extract gait features such as stride length and stride duration. Given the motor anomalies of the parkinsonian gait, we propose features to capture the postural control variability such as the stance phase percentage, or features to describe the coordination and symmetry of the gait such as the duration ratios between left and right limbs.

(2) Moreover, we introduce and discuss new frequency-based features to assess the gait prior to FoG, such as dominant frequency, dominant frequency amplitude and the inverse of the dominant frequency slope of the acceleration data. These features are a quantification of the gait quality.

(3) We report a constant variation of features with few seconds prior to FoG, the most common being the changes in stride duration, stride length, dominant frequency and the inverse of the dominant frequency slope.

To our knowledge, our study is the first to target a continu- 
ous monitoring of gait parameters in the transient between walking and FoG, and compare it with the gait changes before turns. The motivation is the real-time prediction of such episodes using data from wearable sensors.

Our work is structured as follows: In Section 2 we survey the clinical observations of FoG patterns and the use of wearable sensors to describe the gait.We describe our dataset in Section 3, and our experimental framework in Section 4. In Section 5 we present and discuss the features and the potential of predicting FoG by observing trends in the gait properties. We conclude our work in Section 6 .

\section{RELATED WORK}

In the following we review the literature (1) on clinical observations regarding gait patterns correlated with FoG, and (2) on the use of wearable sensors for gait analysis.

Clinical observations of gait patterns prior to FoG. The FoG pathogenesis is not understood at the moment [19, 10]. Nonetheless, there are several gait impairments associated with PD and FoG $[12,23]$ with respect to a healthy person's gait. Among gait anomalies which might contribute to FoG are the stride length reduction [4], step festination [10], deteriorations in rhythmic control $[4,8]$ and in step-tostep time variability [8], or the reduction of the cadence [4, 10]. Plotnik et al. [20] show that the impairments in leftright coordination are associated with the presence of FoG, while Yogev et al. [26] finds that gait asymmetry is increased in people with PD and FoG. Prior to freezing, subjects experience postural instability, which might be compensated by an increased time in which both feet are on the ground [17]. Yet, these evidences are found by comparing groups of subjects with FoG with groups of healthy subjects and people with PD, but who did not experience FoG. Different from prior studies, we focus on observing changes in the gait during the transient period before the FoG episode. These changes, if any, will open the direction of real-time prediction of gait freezing.

To the best of our knowledge, only Nieuwboer and colleagues [17] use gait characteristics to analyze the relation between walking pattern and the gait prior to FoG. The study focuses on the relation between cadence and stride length during normal walking, gait festination, pre-stop, and prior to freeze periods. However, it takes into account only three strides for each of the four groups of walking, for subjects which are in the OFF medication state. Different and in extension to [17], we analyze the gait during the whole transient period from normal walking up to the initiation of FoG or until the initiation of a turn, and extend the number of analyzed gait features. In addition, we use data from patients in ON medication, which makes it more difficult to extract the gait parameters [10], but is more realistic for the case of out-ofthe-lab FoG prediction setting.

Wearable sensors and features for gait analysis. Wearable sensors are used to distinguish kinematic properties, with applications in sports [22], or in healthcare sector for clinical diagnosis or rehabilitation [25]. A wide range of sensors are used to extract gait properties, such as inertial measurement units [22, 25], reflective markers [17] or pressure insoles $[4,8,20]$, usually attached on the lower body.

Characterizing Parkinsonian gait with accelerometers is more complex than in case of healthy subjects, due to the disease nature, which manifests into a large spectrum of motor anomalies [24]. Han and colleagues [7] propose an algorithm based on the a comparation of the standard deviation of each accelerometer data axis with a threshold to distinguish between stance and swing phases in PD. In our work, we use data from 3D wearable accelerometers and gyroscopes attached to the ankle to characterize the parkinsonian kinematics prior to the freezing episodes.

In a previous work, we study the change of time-series features and of unsupervised extracted features from acceleration in DAPHnet dataset [2], in the period prior to FoG events [13]. Although some vary prior and during FoG, such features seem to have limitations in capturing the gait variations prior to FoG, a reason being the unlabeled walking context in the dataset. Gait variability can be represented also using acceleration frequency-based features like the Amplitude [psd], width $[\mathrm{Hz}]$ and slope $[\mathrm{psd} / \mathrm{Hz}]$ of the dominant frequency $[\mathrm{Hz}]$. Weiss et al. [24] show that a larger width and a lower amplitude of the dominant frequency on the locomotion band $[0.5,3] \mathrm{Hz}$ is directly related to the stride-tostride variability and a relative less consistent gait pattern in $\mathrm{PD}$ patients compared with healthy people. We extend these frequency-based features to the total power band of locomotion and freeze $[0.5,8] \mathrm{Hz}[15]$ and propose the inverse of the dominant frequency slope to analyze the spectrum prior to FoG compared with walking and gait prior to turns.

\section{DATASET}

To observe whether gait properties change prior to gait freeze, we extract features from a subset of subjects from Cupid dataset. Cupid [14] was collected with the purpose to describe and characterize FoG from a multimodal sensing perspective, i.e., IMU, skin response, electrocardiogram. Participants performed a clinical protocol in a lab-setting, designed to provoke FoG and simulate daily-life scenarios athome, as for example tasks including passing narrow corridors, turnings, or walking with cognitive load.

We use the information provided by the wearable IMU attached on both ankles of the subjects, sampling synchronized $3 \mathrm{D}$ acceleration, 3D rotation, and 3D magnetometer data at $128 \mathrm{~Hz}$. Data streams from the 2 IMU are synchronized with videos used to provide FoG labels by two clinicians. They considered the moment of the arrested gait pattern, i.e., stop in alternating left-right stepping, as the start of FoG, and the instant when the patient resumed a regular gait pattern as the end of it. Besides FoG, clinicians labeled the start of other walking events, i.e., gait initiation, turns, stops.

From the 18 participants, 7 did not experience freeze during the protocol, while 4 of them had less than 6 FoG, and for two subjects most of FoG happened similar in a chain reaction - freeze events were few seconds apart from each other during walking. We use data from the 5 participants which had more than 10 FoG episodes during the protocol. Included subjects are between 49 and 89 years old, with a disease duration ranging from 3 to 14 years, representing a wide range of PD stages in terms of Hoehn and Yahr score (II to IV) [9]. Subjects were in the ON medication state during protocol, as we aimed for a realistic representation of the usual daily-life setting. 


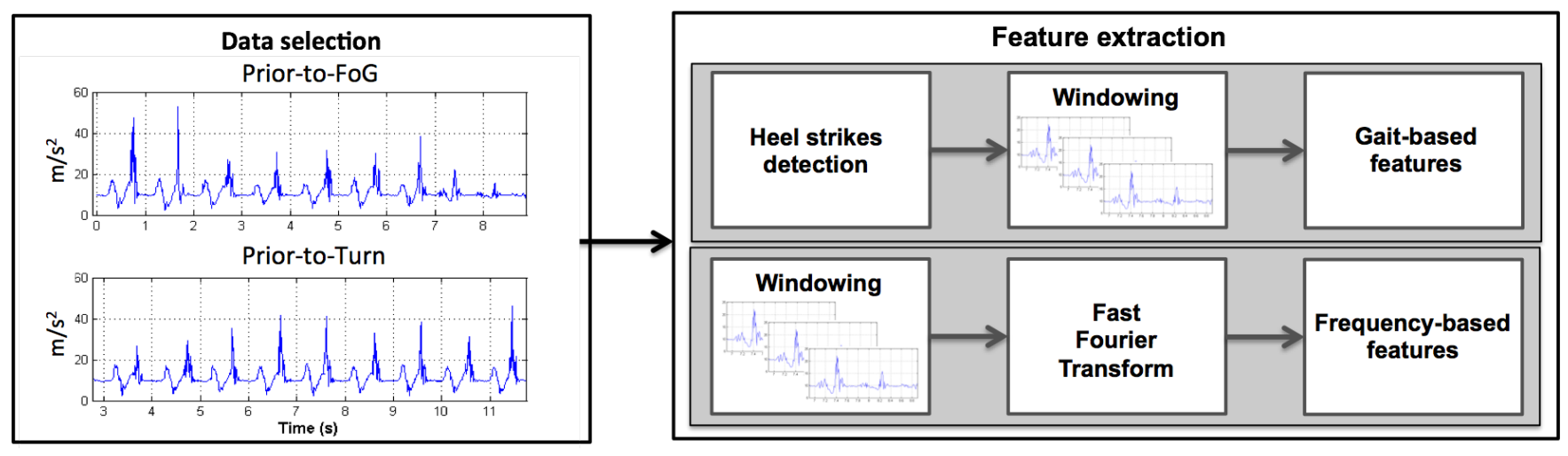

Figure 1: The data analysis framework: In the first phase we select the IMU data from prior-to-FoG and prior-to-turn sessions for each of the 5 subjects. Then, for each session we follow different procedures in case of the two types of features: (1) For gait parameters, we perform a heel strike detection, then we separate the session into 2 seconds non-overlapping windows. From each window we the extract the features. (2) For frequency features, we separate the session into 4 seconds overlapping windows, with a window-overlapping step of 2 seconds, on which we perform a Fourier Transform, and extract the frequency properties.

\section{EXPERIMENTAL SETTING}

In the following, we describe the methodology and the features we extract from IMU to observe whether there is a transition of the gait towards FoG.

\subsection{Methodology}

Figure 1 contains the main steps of our experimental setting: We first consider for each of the 5 subjects only the FoG events during turns and straight line walking. For the FoG during gait initiation, no prior information about the gait degeneration could be extracted, as patients were sitting or standing. Then for each freezing episode, we consider the walking IMU data just prior to it in a straight line, which does not contain any other gait event, such as stop walking, turns or other FoG. We call this period as the prior-toFo $G$ session. To have enough prior-to-FoG information, we keep only sessions which have at least 4 seconds of straight line walking prior to FoG. This duration was selected as a trade-off between having a statistical relevant number of prior-to-FoG sessions, and to have enough information to characterize the gait before FoG.

Similarly to the prior-to-Fo $G$ sessions, we consider for each subject an equal number of prior-to-turn sessions: Walking data prior to a turn is selected in the same manner as prior to a FoG. We use these prior-to-turn information in order to observe whether the gait features before a FoG change in a different manner than before an usual turn.

In a second step, we follow different procedures for the two types of features we extract from the IMU: (a) gait-based features, and (b) frequency-based features. In case of (a) gait properties, for each prior-to-FoG and prior-to-turn session, we detect the gait cycles from the IMU raw data. A gait cycle is defined as the difference between two consecutive heel strike time of the same leg. The heel strike is the moment in which the heel touches the ground and is represented by a maximum peak on the accelerometer magnitude data $[22,25,1]$. In the parkinsonian gait, the heel strike is often confused with the start of the swing phase, due to the walking abnormalities of the disease [25]. In order to avoid false heel strike detection, we use the gyroscope signal to

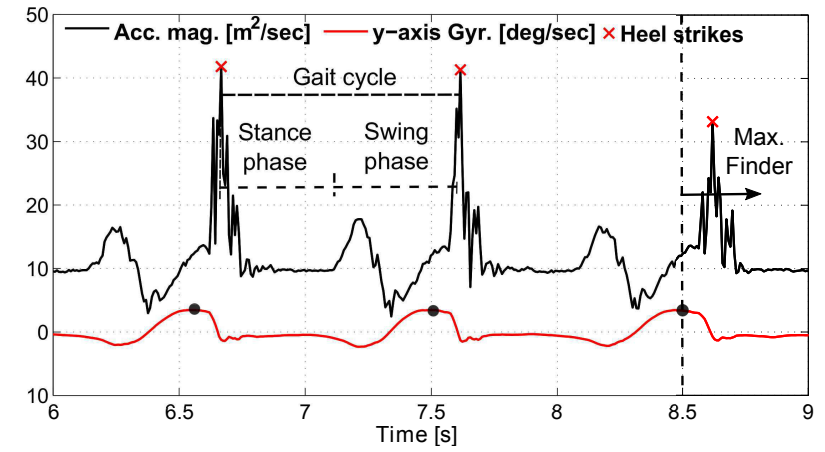

Figure 2: The procedure to detect the heel strike using accelerometers and gyroscopes: First we detect the ending of the swing phase represented as a peak in the gyroscope information. Then we look in the next period of acceleration data to detect the peak in acceleration corresponding to the heel strike.

detect the end of the swing phase, and thus to differentiate between the swing peak and the heel strike as in Figure 2: We first detect the ending of the swing phase represented as a peak in the y-axis gyroscope, and then we look in the next period for a maximum peak detection over the accelerometer magnitude data, which represents the heel strike. However, in some of the sessions the subjects experience the so-called foot drop, in which the swing phase is no present and can not be detected prior to FoG. These result in false detection or even no detection of the heel strike. In these rare cases, we manually set the heel strike.

Once the gait cycles are extracted, we separate each session of data in non-overlapping windows of $N_{\text {window }}=2$ seconds. A full gait cycle lasts for 1-1.5 seconds, thus a window of 2 seconds is enough to capture it and to extract the gait parameters as detailed in Section 4.2.

In case of the (b) frequency features, we first separate each session of raw IMU data in overlapping windows of $N_{\text {window }}=$ 4 seconds with an overlapping step of $N_{\text {overlapp }}=2$ seconds. We choose the window length as a trade-off between 

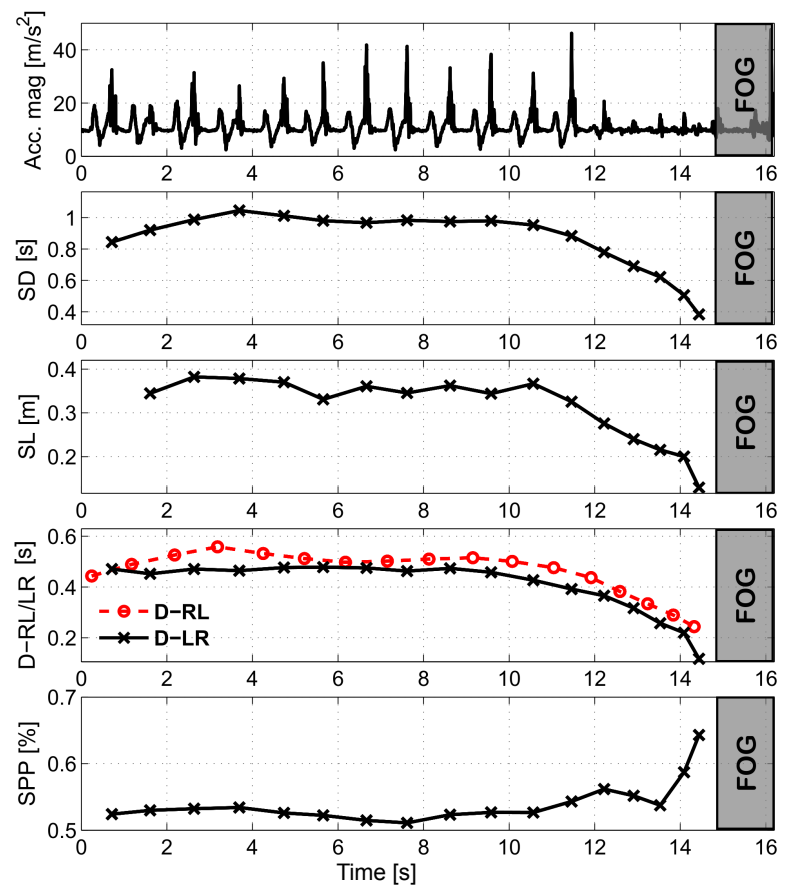

Figure 3: A prior-to-FoG session, expressed in raw data from acceleration and from gait parameters. We observe that with 2 and 4 seconds prior to FoG, the stride duration, stride length and both D-RL and D-LR decrease, while SPP increases. However, the gait features tend to remain constant during straight line walking (up to 10 seconds).

the latency of observations related to the upcoming FoG and the FFT estimation error. On each window we perform a Fast Fourier Transformation (FFT) on the magnitude vector extracted from the raw 3D acceleration data. We then compute the frequency features detailed in Section 4.3.

\subsection{Gait Features}

We extract the following gait-based features:

(1) Stride duration (SD): The time between two consecutive steps of the same limb. We compute SD as the time difference between two consecutive detected acceleration peaks of the same leg.

(2) Stride length (SL): The covered distance between two consecutive steps of the same limb. We consider SL as the double integration of the acceleration signal according to the sampling rate, between two consecutive acceleration peaks of the same limb.

(3) Right-Left Duration (D-RL)/ Left-Right Duration (D-LR): The time between a right heel peak and the consecutive left heel peak (D-RL), or the left heel and the consecutive right heel peaks (D-LR). Both D-RL and D$\mathrm{LR}$, are a measure of the bilateral coordination and the gait asymmetry/rhythmicity of the patient.

(4) Stance Phase Percentage (SPP) [\%]: The time in which the foot is in contact with the ground over the whole stride duration of the same limb. D-RL can be considered as the time the left foot is in contact with the ground (stance time) and the time the right foot is in the air (swing time).
The relation between D-LR and the stride duration of the right leg gives the stance phase percentage of the right leg. This feature is a measure of the postural control in PD [26].

As an example, in Figure 3 we plot the raw acceleration data from a prior-to-FoG session, together with the extracted gait features. In the first 10 seconds, there are no high variations in the gait features. However, with 2 to 4 seconds prior to FoG, the SD, SL, D-RL and D-LR abruptly decrease, while the stance phase percentage increases.

\subsection{Frequency-based Features}

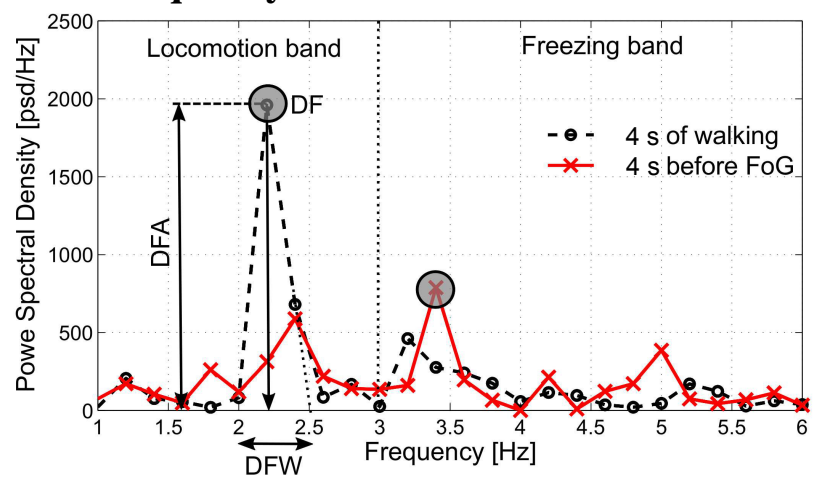

Figure 4: The acceleration's power spectral density between $[1,6] \mathrm{Hz}$, from a 4-second window just prior to FoG, and from a 4 -second window during mere walking, from the same prior-to-FoG session. The three frequency features are different just prior to FoG, compared with walking: DF migrates to $[3,8]$ $\mathrm{Hz}$ freeze band, while its amplitude decreases, and the relation width-amplitude increases prior to FoG.

Most of the energy during walking in healthy subjects concentrates over $[0.5,3] \mathrm{Hz}$ range around a dominant frequency and with a high amplitude. FoG episodes are related with gait asymmetry, suggesting variations of the walking frequency, while the energy might spread over a larger range of frequencies. Therefore a decrease in the dominant frequency amplitude and an increase in its width might be an indicator of gait symmetry variations [24]. Moreover, the $\mathrm{SD}$ is directly related to the walking frequency. Hence, a reduction of the stride duration might be also related to an increase of the dominant frequency.

We propose the following frequency features to characterize the gait variability prior to FoG:

(1) Dominant Frequency (DF) $[\mathrm{Hz}]$ : The frequency with the highest energy in the $[0.5,8] \mathrm{Hz}$ band, which sums both normal gait and FoG frequency bands.

(2) Dominant Frequency Amplitude (DFA) [psd]: Corresponds to the power of the dominant frequency.

(3) Inverse of the Dominant Frequency Slope (IDFS) $[\mathrm{Hz} / \mathrm{psd}]$ : The relation between the dominant frequency width (DFW) and the DFA where the DFW is the frequency range of the DF obtained through the extension of the DF's slopes.

Figure 4 shows the power spectral density with respect to the frequency, on a 4 seconds window of straight line walking acceleration, and on the 4 seconds window just prior to a FoG episode, for the same subject. We observe that with 4 seconds just prior to FoG the dominant frequency migrates 

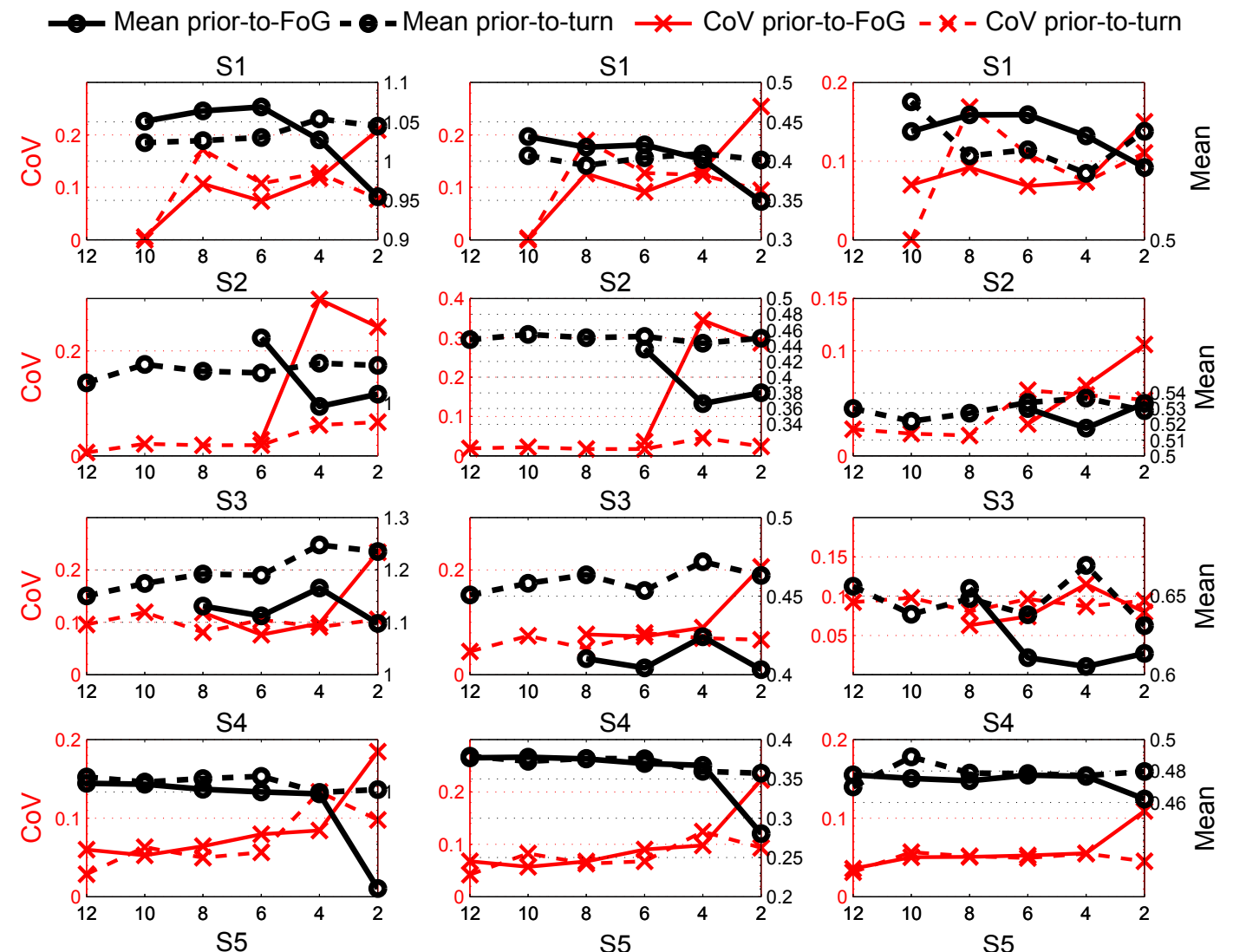

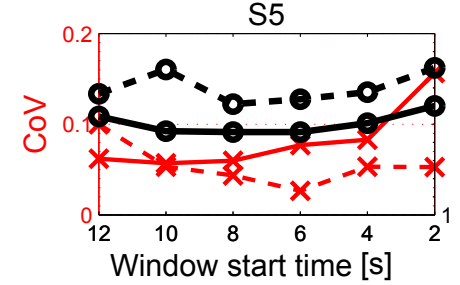

(a) SD (s)

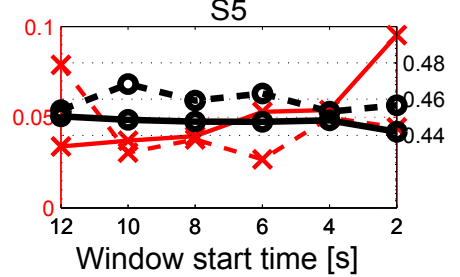

(b) SL [m]

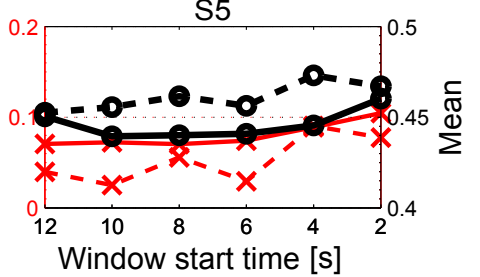

(c) SPP [\%]

Figure 5: The mean and CoV values in case of stride duration, stride length, and stance phase percentage for the two different types of walking sessions, in each of the 5 subjects data. The numbers of the time axis represent the time difference between the start of FoG and the start of the window from which features were computed. For example, 2 seconds means that features were computed in the data window that started with 2 seconds prior-to-FoG, 4 seconds - in the window which started with 4 seconds prior to FoG, and so on.

to the so-called freeze band, being higher than $3 \mathrm{~Hz}$, while in case of the 4 seconds window of normal walking, the DF is in the range of the gait movement $[0.5,3] \mathrm{Hz}$. Moreover, the DFA decrease just prior to FoG, compared with walking.

\section{ANALYSIS AND DISCUSSION}

To observe whether there are general trends in gait and frequency features prior to FoG, for each of the 5 subjects, we compute statistics such as mean, standard deviation, and the coefficient of variation overall the prior-to-Fo $G$ sessions. The statistics are computed for each synchronized window, as for example in windows which start 2 seconds prior to FoG, or start with 4 seconds prior FoG, and so on. The coefficient of variation $(\mathrm{CoV})$ is defined as the ratio of the standard deviation to the mean, and in our case is a measure of how much dispersed are the the values of a specific feature in a window. To determine if the changes in the features are specific prior to FoG, we apply the same methodology for the features in each window from all prior-to-turn sessions of the same subject. In the following, we discuss our findings with respect to the two categories of features extracted.

\subsection{Gait Features Trends prior to FoG}

Figure 5 shows the $\mathrm{CoV}$ and mean statistics for each of the 5 subjects, in case of $\mathrm{SD}, \mathrm{SL}$, and $\mathrm{SPP}$ gait features. We will discuss further the results for each of them:

Stride duration. Clinical literature relates FoG with a decrease of in the SD $[4,17,10]$ in subjects with FoG compared with subjects without FoG or healthy ones. We observe from Figure 5 that for all the 5 subjects, the $\mathrm{CoV}$ increases just prior to FoG, showing a higher dispersion of the stride duration in windows starting with 2 to 4 seconds prior to FoG. While for the rest of the walking session, the $\mathrm{CoV}$ values tend to have constant values across all windows (except in case of $\mathrm{S} 2$, on which we did not have sessions 
longer than 6 seconds prior-to-FoG). Moreover, when compared with prior-to-turn information, we observe that the $\mathrm{CoV}$ values tend to be constant across all the window times, except in case of $\mathrm{S} 4$, where the $\mathrm{CoV}$ also increases prior to turn, but the variation is smaller than in the case of FoG.

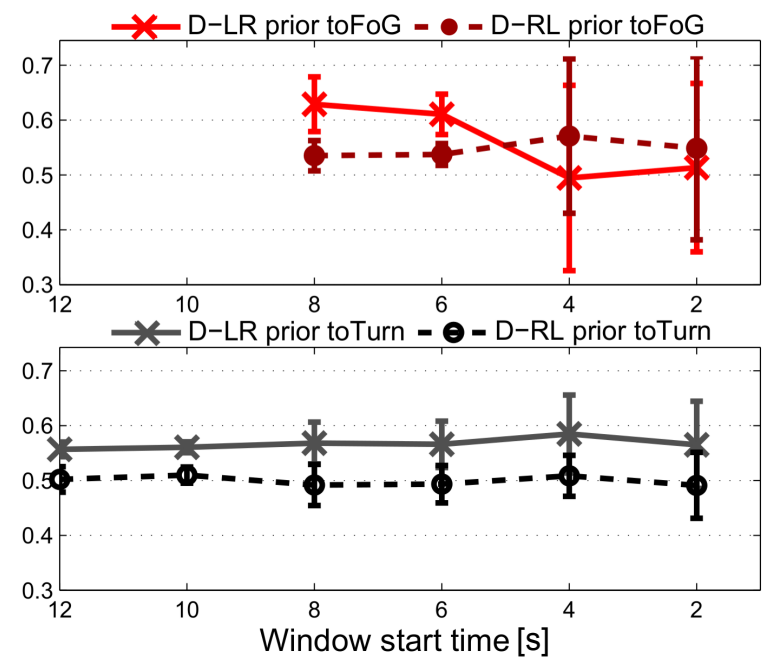

Figure 6: The variation of D-LR and D-RL features in case of prior-to-FoG and prior-to-turn sessions for S2. The values on the time axis follow the same convention as in Figure 5. For walking prior to turn, their average values and the ratio remains constant, and in windows starting with 4 seconds before FoG the D-LR decreases, while D-RL stays constant, suggesting gait asymmetry just prior to FoG.

Even if the $\mathrm{CoV}$ always increases in windows with few seconds prior to FoG, the SD follows different trends across datasets: For the first 4 subjects, the mean stride duration decreases with 2-4 seconds windows prior to FoG, with an overall average values between 0.1-0.2 seconds, while for S5 SD slightly increases with approx. 0.05 seconds, but also this happens in the periods prior to turns. In case of S3, even if the SD decreases with 2 second prior to turn, first it increases with up to 0.1 seconds with 4 seconds before turns.

Stride length. The same observations as for SD are present also in case of the SL feature: The CoV increases for all 5 subject in data up to 4 seconds of data before FoG, suggesting a high dispersion of the stride length values prior to FoG. SD and SL are directly related: In case of S1, S2 and S4 the mean of SL decreases with an average between 0.1 and $0.05 \mathrm{~m}$ in the windows starting 2 to 4 seconds just before FoG, compared with all the rest of walking. However, in case of S5 the mean stride length also slightly decreases with approx. average of $0.02 \mathrm{~m}$, even if the SD increases, in this case suggesting stepping hesitation that might cause an eventual FoG. For all subjects SL tends to remain constant prior-to-turns.

For S3 and S5, even if prior to FoG the SL changes only slightly, when comparing prior-to-Fo G SL with prior-to-turn SL values, we observe that constantly all over the session the SL prior to turn is lower than in the case of mere walking with turns (with up to $0.05 \mathrm{~m}$ for S3 and a constant 0.02 $\mathrm{m}$ in case of S5). The same observation holds in the case of SD feature for both subjects.
Stance Phase Percentage. Unlike the previous SD and SL features, the SPP CoV do not follow a clear trend. However, the stance phase percentage varies for $\mathrm{S} 1-\mathrm{S} 4$ patients, in windows starting with 2 seconds (S4) up to 6 seconds (S1 and S3) prior to FoG. SPP is a measure of the postural control, gait asymmetry and rhythmicity. Thus, the variation of SPP prior to FoG, as in case of S1, S3 and S4, suggest that the subjects loose the postural control just prior to FoG, compared to prior to a turn. However, for S1 and S3 the SPP also changes prior to turns, but opposite to the case of FoG sessions. In case of S5, the SPP is constant across time windows, but it is constantly lower than in case of sessions with mere walking and turns. This suggests that for this subject there is an anomaly of walking defined by all three SL, SD and SPP features which might be a cause of FoG, but over long sessions of time.

LR/RL Limb Durations. D-RL and D-LR features are a measure of the gait symmetry. In healthy subjects, D$\mathrm{RL}$ and D-RL tend to have similar values during walking, expressing a symmetrical gait. In case of prior-to-turn session, all subjects tend to have constant ratio for D-RL/D-LR times. In case of prior-to-FoG data in $\mathrm{S} 2$ and $\mathrm{S} 3$, this ratio tends to change and also to have larger ranges towards 2 to 4 seconds before FoG, as shown also in Figure 6 for S2: In case of turns the D-RL/D-LR tends to remain constant, while in case of FoG, there is a variation in D-RL feature from data window starting 4 seconds prior to freeze. This suggests an asymmetry between the left and right limb prior to FoG, which might cause the freeze. However, for the other 3 subjects, this ratio is similar as in the case of turns.

\subsection{Frequency Features Trends prior to FoG}

In case of the three frequency features, the $\mathrm{CoV}$ for each window of walking does not show any significant differences in case of prior-to-FoG and prior-to-turn sessions in any subjects datasets, except for S2. However, In Figure 7, we plot the mean and standard deviation for the three frequency features in prior-to-FoG and prior-to-turn data. We observe that the dominant frequency tends to increase in windows starting 4 up to 6 seconds prior to FoG for all subjects. In case of S1, S2 and S4 the DF even migrates from the normal gait band of $[0.5,3] \mathrm{Hz}$ to the freezing band, being higher than $3 \mathrm{~Hz}$, with a higher variation of the feature values across sessions, compared with the mere walking.

In case of the IDFS feature, it increases in windows starting 4 to 6 seconds prior to FoG, compared with periods of normal walking preceding turns for all subjects. S1, S2 and S3 are particular cases, as DFA is constantly lower in sessions ending with a FoG, than compared with the walking sessions with turns, pointing towards a walking anomaly which lasts longer and might cause eventually a FoG. In this case the cueing can be given when this anomaly is observed, as it can help patients to ameliorate the gait.

\subsection{Discussion}

Table 1 contains a summary of our results regarding the gait variability before FoG, outlining the most informative features across all five subjects.

Stride length, stride duration and the stance phase percentage show significant variations prior to FoG for 4 out of 5 subjects. Moreover, these changes are not present during mere walking prior-to-turns sessions. These significant 

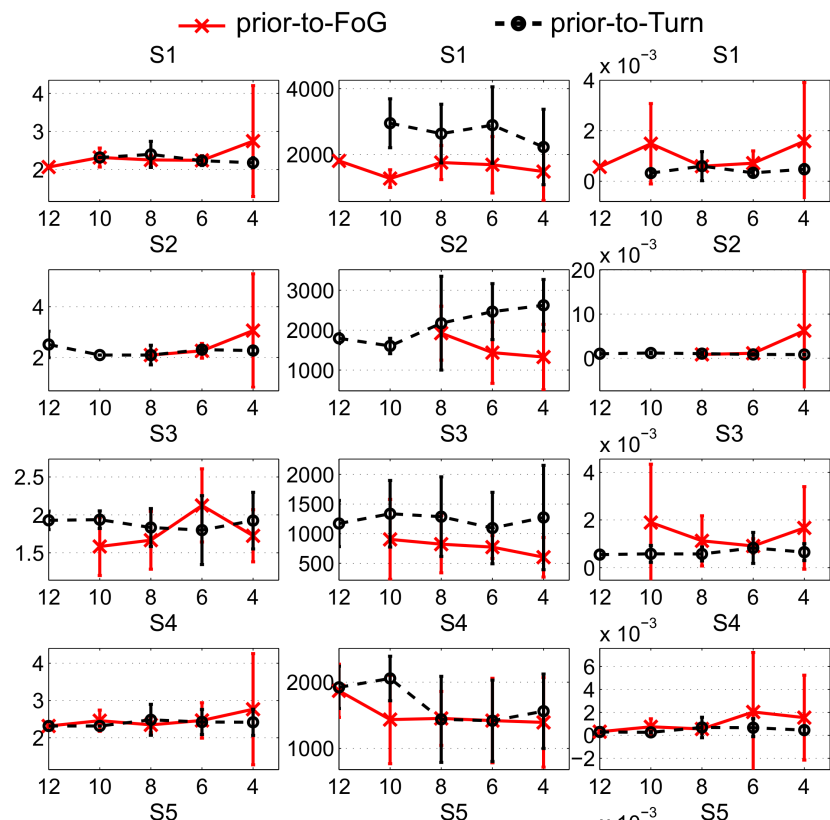

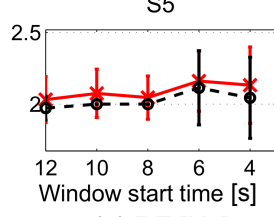

(a) DF $[\mathrm{Hz}]$

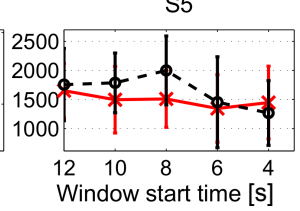

(b) DFA [psd]

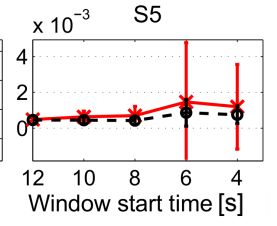

(c) IDFS [Hz/psd]
Figure 7: The average and standard deviation across all 5 subjects, for the three frequency features in case of prior-to-FoG and prior-to-turn. The time axis follows the same convention as in Figure 5.

changes can be observed on average in windows starting 2 to 4 seconds before FoG. In case of S5, even if there are not significant changes in these 3 features with few seconds before FoG, in case of prior-to-FoG sessions the average values for SD, SL, and SPP are constantly lower than in case of prior-to-turn sessions.

These characteristic trends of the SD, SL and SPP prior to FoG open the direction of predicting such freeze episodes with seconds before they are about to happen for S1-S4, and even help the subject correct the anomalous gait which will lead eventually to FoG, as in case of S5. Moreover, these observations are based on periods which transition from mere walking towards FoG, thus making possible a real-time prediction of such freezing episodes.

From the frequency features, the dominant frequency and the inverse of the dominant frequency slope increase in all of 5 subjects with 4 up to 6 seconds prior to FoG and the DF migrates towards the $[3,8] \mathrm{Hz}$ band for 3 out of 5 subjects, which describes the FoG event.

All the other gait features such as D-RL/-LR and frequency features such as DFA are informative and helpful for FoG prediction, but are dependent and specific to some subjects. This suggests that there are anomalies of the gait with few seconds prior to FoG which announce the freeze, but these anomalies are subject specific. For building a model of FoGprediction, we would need to take into account different
Table 1: Informative features across subjects

$\begin{array}{lccccc}\text { Feature } & \text { S1 } & \begin{array}{c}\text { S2 } \\ \text { Gait }\end{array} & \text { Sarameters } & \text { S4 } & \text { S5 } \\ \text { SD } & \checkmark & \checkmark & \checkmark & \checkmark & \text { no } \\ \text { SL } & \checkmark & \checkmark & \checkmark & \checkmark & \text { no } \\ \text { SPP } & \checkmark & \checkmark & \checkmark & \checkmark & \text { no } \\ \text { D-(RL) } & \text { no } & \checkmark & \checkmark & \text { no } & \text { no } \\ \text { DF } & & \text { Frequency } & \text { features } & \\ \text { DFA } & \checkmark & \checkmark & \checkmark & \checkmark & \checkmark \\ \text { IDFS } & \checkmark & \checkmark & \checkmark & \text { no } & \text { no } \\ & \checkmark & \checkmark & \checkmark & \checkmark & \checkmark\end{array}$

sets of gait and frequency features for each subject: Stride length, stride duration, dominant frequency, and the inverse of the dominant frequency slope seem to be a common set, on which specific features such as DFA or SPP can be added, depending on the gait anomalies of the subject, as for example for S5 the gait hesitation prior to FoG, or gait asymmetry in case of $\mathrm{S} 2$.

In this study, we used data from patients in the ON medication state, which might milden the overall gait variations prior to FoG. However, we were able to observe changes in the gait prior to freeze even in these realistic setting, which gives the possibility to predict FoG with a wearable system in a daily-life out-of-the-lab setting, where patients follow their regular PD treatment.

All the gait and frequency features to characterize the gait prior to FoG were extracted from wearable IMU mounted on the ankle of the subjects. There are already wearable systems which use information from on-body attached IMU to detect FoG in real-time and to start cueing to support the user to exit FoG and resume walking $[11,2]$. Such systems can be extended with gait features and methods to predict FoG, given that the same information is used for both tasks.

\section{CONCLUSION}

We analyze the gait in the transient period between walking and freezing of gait in Parkinson's disease, and compare it with periods prior to other walking events such as turns. Our motivation is to find specific gait characteristics which change prior to FoG, thus enabling the idea of predicting in real-time FoG with few seconds before, in order to help the subject avoid the upcoming event.

We use inertial measurements unit mounted on both ankles of $5 \mathrm{PD}$ subjects with freezing of gait. We compute gaitrelated parameters used to monitor the gait in sports and healthcare such as stride length and stride duration. Moreover, we propose specific gait and frequency features to catch the Parkinsonian gait characteristics, such as stance phase duration, dominant frequency of gait, or the inverse of the dominant frequency slope.

Our results suggest that stride length and stride duration significantly changes in all of 5 subjects, and on 4 of them in data starting with 2 up to 4 seconds prior to FoG. The dominant frequency also tends to migrate to the $[3,8] \mathrm{Hz}$ band for 3 subjects with 4 up in data windows starting up 6 seconds prior to FoG and the inverse of the dominant frequency slope increase in the information starting with 4 up to 6 seconds prior to FoG compared with prior-to-turn sessions for all subjects. All other features capture specific gait charac- 
teristics prior to FoG, although are subject-dependent.

These evidences suggest that gait and frequency parameters from IMU might be used to predict FoG with few seconds before it happens. However, these findings are preliminary, due to the limited number of subjects in the experiments. For future work, we consider to increase the number of subjects, and to compare prior-to-FoG to other gait contexts, such as prior-to-stop periods.

\section{REFERENCES}

[1] K. Aminian, B. Najafi, C. Büla, P. Leyvraz, and P. Robert. Spatio-temporal parameters of gait measured by an ambulatory system using miniature gyroscope. Journal of Biomechanics, 35:689-99, 2002.

[2] M. Bächlin, M. Plotnik, D. Roggen, I. Maidan, J. M. Hausdorff, N. Giladi, and G. Tröster. Wearable assistant for Parkinson's disease patients with the freezing of gait symptom. IEEE Transactions on Information Technology in Biomedicine, 2010.

[3] B. R. Bloem, J. M. Hausdorff, J. Visser, and N. Giladi. Falls and freezing of gait in Parkinson's disease: A review of two interconnected, episodic phenomena. Movement Disorders, 19:871-884, 2004.

[4] R. Chee, A. Murphy, M. Danoudis, N. Georgiou-Karistianis, and R. Iansek. Gait freezing in Parkinson's disease and the stride length sequence effect interaction. Brain, 132:2151-2160, 2009.

[5] S. Donovan, C. Lim, N. Diaz, N. Browner, P. Rose, L. Sudarsky, D. Tarsy, S. Fahn, and D. Simon. Laserlight cues for gait freezing in Parkinson's disease: an open-label study. Parkinsonism \& Related Disorders, 17:240-245, 2011.

[6] P. Gray and K. Hildebrand. Fall risk factors in Parkinson's disease. Journal of Neuroscience Nursing, 32(4):222-228, 2000.

[7] J. Han, H. Jeon, and K. Park. Gait detection from three dimensional acceleration signals of ankles for patients with parkinson's disease. Proc. International Special Topic Conference on Information Technology in Biomedicine, 2006.

[8] J. Hausdorff, J. Schaafsma, Y. Balash, B. A.L., T. Gurevich, and N. Giladi. Impaired regulation of stride variability in Parkinson's disease subjects with Freezing of Gait. Experimental Brain Research, 149:187-194, 2003

[9] M. Hoehn and M. Yahr. Parkinsonism: onset, progression and mortality. Neurology, 50(2):318, 1998.

[10] R. Iansek, F. Huxham, and J. McGinley. The sequence effect and gait festination in Parkinson disease: contributors to freezing of gait? Mov Disorders, 21:1419-1424, 2006.

[11] E. Jovanov, E. Wang, L. Verhagen, M. Fredrickson, and R. Fratangelo. deFOG: A real time system for detection and unfreezing of gait of Parkinson's patients. In Engineering in Medicine and Biology Society. Annual International Conference of the IEEE, pages 5151-4, 2009.

[12] S. Lord, K. Baker, A. Nieuwboer, D. Burn, and L. Rochester. Gait variability in Parkinson's disease: an indicator of non-dopaminergic contributors to gait dysfunction? J Neurology, 258:566-572, 2011.

[13] S. Mazilu, A. Calatroni, E. Gazit, D. Roggen, J. M.
Hausdorff, and G. Tröster. Feature learning for detection and prediction of freezing of gait in Parkinson's disease. In Machine Learning and Data Mining in Pattern Recognition, pages 144-158, 2013.

[14] S. Mazilu, E. Gazit, U. Blanke, D. Roggen, J. M. Hausdorff, and G. Tröster. Engineers meet clinicians: augmenting Parkinson's disease patients to gather information for gait rehabilitation. In Proceedings of the 4th Augmented Human International Conference, pages 124-127. ACM, 2013.

[15] S. Moore, H. MacDougall, and W. Ondo. Ambulatory monitoring of freezing of gait in Parkinson's disease. Journal of Neuroscience Methods, 167:340-348, 2008.

[16] W. Muangpaisan, A. Mathews, H. Hori, and D. Seidel. A systematic review of the worldwide prevalence and incidence of Parkinson's disease. Journal of the Medical Association of Thailand, 94(6):749-55, 2011.

[17] A. Nieuwboer, R. Dom, W. De Weerdt, et al. Abnormalities of the spatiotemporal characteristics of gait at the onset of freezing in Parkinson's disease. Movement Disorders, 16:1066-1075, 2001.

[18] A. Nieuwboer, G. Kwakkel, L. Rochester, D. Jones, et al. Cueing training in the home improves gait-related mobility in Parkinson's disease: the RESCUE trial. Journal of Neurology, Neurosurgery and Psychiatry, 78(2):134-140, 2007.

[19] J. G. Nutt, B. R. Bloem, N. Giladi, M. Hallett, F. B. Horak, and A. Nieuwboer. Freezing of gait: moving forward on a mysterious clinical phenomenon. Lancet Neurolology, 10:734-744, 2011.

[20] M. Plotnik, N. Giladi, and J. Hausdorff. Bilateral coordination of walking and freezing of gait in Parkinson's Disease. European Journal of Neuroscience, 27(8):1999-2006, 2008.

[21] M. Plotnik, N. Giladi, and J. Hausdorff. Is Freezing of Gait in Parkinson's Disease a result of multiple gait impairments? Implications for treatment. Parkinson's Disease, 2012.

[22] C. Strohrmann, H. Harms, C. Setz, and G. Troester. Monitoring kinematic changes with fatigue in running using body-worn sensors. IEEE Transactions on Information Technology in Biomedicine, 16(5):983-990, 2012.

[23] J. Vandenbossche, N. Deroost, E. Soetens, J. Spildooren, S. Vercruysse, A. Nieuwboer, and E. Kerckhofs. Freezing of gait in Parkinson Disease is associated with impaired conflict resolution. Neurorehabil Neural Repair, 25(8):765-773, 2011.

[24] A. Weiss, S. Sharifi, M. Plotnik, J. van Vugt, N. Giladi, and J. Hausdorff. Towards automated, at-home assessment of mobility among patients with Parkinson Disease, using a body-worn accelerometer. Neurorehabil Neural Repair, 25(9):810-818, 2011.

[25] A. Willemsen, F. Bloemhof, and H. Boom. Automatic stance-swing phase detection from accelerometer data for peronel nerve stimulation. IEEE Transactions on Biomedical Engineering, 37(12):1201-8, 1990.

[26] G. Yogev, M. Plotnik, C. Peretz, N. Giladi, and J. Hausdorff. Gait asymmetry in patients with Parkinson's disease and elderly fallers: when does the bilateral coordination of gait require attention? Exp Brain Res, 177(3):336-346, 2007. 